\title{
Effects of insulin and exercise training on FGF21, its receptors and target genes in obesity and type 2 diabetes
}

\author{
Rikke Kruse $^{1,2,3}$ - Sara G. Vienberg ${ }^{4}$ - Birgitte F. Vind ${ }^{3}$ - Birgitte Andersen ${ }^{4}$. \\ Kurt Højlund ${ }^{1,2,3}$
}

Received: 13 March 2017 / Accepted: 9 June 2017 / Published online: 18 July 2017

(C) Springer-Verlag GmbH Germany 2017

\begin{abstract}
Aims/hypothesis Pharmacological doses of FGF21 improve glucose tolerance, lipid metabolism and energy expenditure in rodents. Induced expression and secretion of FGF21 from muscle may increase browning of white adipose tissue (WAT) in a myokine-like manner. Recent studies have reported that insulin and exercise increase FGF21 in plasma. Obesity and type 2 diabetes are potentially FGF21-resistant states, but to what extent FGF21 responses to insulin and exercise training are preserved, and whether FGF21, its receptors and target genes are altered, remains to be established.

Methods The effects of insulin during euglycaemichyperinsulinaemic clamps and 10 week endurance training on serum FGF21 were examined in individuals with type 2 diabetes and in glucose tolerant overweight/obese and lean individuals. Gene expression of FGF21, its receptors and target genes in muscle and WAT biopsies was evaluated by quantitative real-time PCR (qPCR).

Results Insulin increased serum and muscle FGF21 independent of overweight/obesity or type 2 diabetes, and there were
\end{abstract}

Electronic supplementary material The online version of this article (doi:10.1007/s00125-017-4373-5) contains peer-reviewed but unedited supplementary material, which is available to authorised users.

Kurt Højlund

kurt.hoejlund@rsyd.dk

1 Department of Clinical Research, Section of Molecular Diabetes and Metabolism, University of Southern Denmark, Odense, Denmark

2 Department of Molecular Medicine, Section of Molecular Diabetes and Metabolism, University of Southern Denmark, Odense, Denmark

3 Department of Endocrinology, Odense University Hospital, Kløvervænget 6, DK-5000 Odense, Denmark

4 Diabetes Research Unit, Novo A/S, Måløv, Denmark no effects associated with exercise training. The insulininduced increases in serum FGF21 and muscle FGF21 expression correlated tightly $(p<0.001)$. In WAT, overweight/obesity with and without type 2 diabetes led to reduced expression of $K L B$, but increased FGFRIc expression. However, the expression of most FGF21 target genes was unaltered except for reduced CIDEA expression in individuals with type 2 diabetes. Conclusions/interpretation Insulin-induced expression of muscle FGF21 correlates strongly with a rise in serum FGF21, and this response appears intact in overweight/ obesity and type 2 diabetes. FGF21 resistance may involve reduced $K L B$ expression in WAT. However, increased FGFR1c expression or other mechanisms seem to ensure adequate expression of most FGF21 target genes in WAT.

Keywords Adipose tissue $\cdot$ FGF21 - Skeletal muscle

$\begin{array}{ll}\text { Abbreviations } \\ \text { ATF4 } & \text { Activating transcription factor } 4 \\ \text { FGF } & \text { Fibroblast growth factor } \\ \text { FGFR } & \text { Fibroblast growth factor receptor } \\ \text { GDR } & \text { Glucose disposal rate } \\ \text { qPCR } & \text { Quantitative real-time PCR } \\ \text { sSDNA } & \text { Single-stranded DNA } \\ \text { WAT } & \text { White adipose tissue }\end{array}$

\section{Introduction}

Fibroblast growth factor (FGF) 21 was originally identified as a metabolic regulator in high throughput screening for agents capable of increasing glucose uptake in adipocytes and has been characterised as a potent endocrine metabolic regulator [1]. Administration of pharmacological doses of FGF21 
improves plasma glucose and lipid profiles, and leads to weight loss in obese diabetic rodents and rhesus monkeys [1-6], possibly due to increased total energy expenditure and physical activity levels [5]. Besides increasing glucose uptake, FGF21 may inhibit lipolysis in white adipose tissue (WAT) and may also promote 'browning' of WAT in response to cold exposure and under other conditions [7-9]. Additionally, a pharmacological dose of FGF21 has been found to reverse palmitate-induced insulin resistance in human myotubes [10] and to improve glucose uptake in skeletal muscle and the heart to normal levels in mice with diet-induced obesity [5].

Prolonged fasting has been shown to increase hepatic FGF21 production and secretion in a PPAR $\alpha$-dependent manner $[11,12]$. FGF21 stimulates fatty acid oxidation, ketogenesis and gluconeogenesis, while it suppresses lipogenesis in the liver [11-13]. During fasting and diet-induced obesity, circulating FGF21 is believed to be completely derived from the liver [14]. Circulating FGF21 mediates its effects through interactions with a dual receptor complex consisting of the cofactor $\beta$-Klotho and a tyrosine kinase FGF receptor (FGFR) $[15,16]$; of which FGFR1c appears to play the predominant role [15]. While the expression of $\beta$-Klotho is limited to a few metabolic tissues, including adipose tissue [17, 18], FGFR1c has a more broad expression pattern [18]. Thus, besides increasing the receptor-affinity for FGF21, $\beta$-Klotho may define its target tissues [16].

Skeletal muscle is not considered a main site of FGF21 expression and release [18]. However, recent studies have reported that muscle stress increases expression of FGF21 [9, 19], possibly in an activating transcription factor 4 (ATF4)-dependent manner [9], and that muscle-derived FGF21 promotes 'browning' of WAT supporting its potential role as a myokine [9]. Moreover, studies in mice and human myotubes have shown an insulin-mediated induction and potential release of FGF21 $[20,21]$. These observations are supported by human studies showing increased $F G F 21$ expression in skeletal muscle and increased circulating FGF21 in response to insulin in healthy young men $[22,23]$. Whether insulin resistance in obese and type 2 diabetic individuals attenuates the insulin-mediated regulation of FGF21 in muscle and plasma has, to our knowledge, not yet been determined.

Exercise training improves whole body glucose homeostasis and reduces the risk of developing type 2 diabetes [24,25]. Given that FGF21 may be released from muscle in mice and that it exerts its beneficial effects by reducing fat mass and enhancing energy expenditure [4], its metabolic effects resemble those of other exercise-induced myokines, such as IL-6, IL-15 and irisin [26]. Acute exercise increases plasma FGF21 in both mice and humans [27]. Furthermore, in humans a transient exercise-induced increase in plasma FGF21 was related to augmented release of FGF21 from the liver in a glucagon-to-insulin-ratio-dependent manner [28], a response that was attenuated in individuals with type 2 diabetes [29]. In contrast, the reported effects of exercise training on circulating FGF21 in humans are variable [30-33]. Thus, long-term training interventions may reduce circulating FGF21 in obese women and elderly men $[30,33]$. However, the effect of exercise training on basal and insulin-stimulated serum FGF21 in individuals with type 2 diabetes remains to be established.

Despite promising studies on the metabolic effects of FGF21 in rodents, the physiological role of FGF21 in humans remains poorly understood. Serum FGF21 is highly variable in humans $[32,34]$ and, paradoxically, it is increased in individuals with obesity, the metabolic syndrome and type 2 diabetes [21, 35-38]. Obesity has been proposed as a state of FGF21 resistance since the response to exogenous FGF21 is impaired in obese mice [39]. This is supported by reduced expression of the FGF21 receptors FGFR1c, FGFR2 and $F G F R 3$, and the co-receptor $\beta$-Klotho (encoded by $K L B$ ) in WAT of obese mice [39]. Additionally, the $\beta$-Klotho transcript and protein is reduced in human adipose tissue in obesity [37]. It remains to be clarified whether FGF21 resistance can be explained by altered mRNA levels of the FGFRs, $\beta$-Klotho, and known target genes in muscle and subcutaneous WAT of individuals with obesity and type 2 diabetes.

In the present study, we examined: (1) whether insulin resistance in obesity and type 2 diabetes attenuates insulinmediated regulation of FGF21 in muscle and serum; (2) how exercise training affects circulating FGF2 1 in type 2 diabetes; and (3) whether FGF21 resistance can be explained by changes in FGFRs, $\beta$-Klotho, and known target genes in muscle and fat in obesity and type 2 diabetes.

\section{Methods}

Participants The effects of insulin and exercise training were studied in blood and tissue samples collected in two prior studies from which other results have been reported [25, 40-44]. The effect of insulin was studied in ten overweight/obese individuals with type 2 diabetes group-wise matched for age and sex to 12 healthy lean and ten overweight/obese non-diabetic volunteers (study I, Table 1). The effect of 10 weeks of endurance exercise training was studied in 12 overweight/obese men with type 2 diabetes and 12 overweight/obese glucose tolerant control men who were group-wise matched for age and obesity (study II, electronic supplementary material [ESM] Table 1). For more information on the study participants, please refer to ESM Methods. All participants gave written informed consent and the studies were approved by the Local Ethics Committee of Funen and Vejle County, Denmark. The studies were performed in accordance with the Helsinki Declaration.

Euglycaemic-hyperinsulinaemic clamp The participants in both studies underwent a euglycaemic-hyperinsulinaemic clamp with tracer glucose $\left(3-\mathrm{H}^{3}\right.$-glucose) to assess total glucose 
Table 1 Metabolic characteristics for participants in study I

\begin{tabular}{|c|c|c|c|}
\hline Characteristic & Lean & Overweight/obese & Type 2 diabetes \\
\hline$n$ (female/male) & $12(6 / 6)$ & $10(4 / 6)$ & $10(6 / 4)$ \\
\hline Age (years) & $54.5 \pm 1.3$ & $55.4 \pm 1.2$ & $53.9 \pm 1.6$ \\
\hline Body weight (kg) & $71.1 \pm 3.5$ & $93.3 \pm 4.8$ & $83.8 \pm 5.0^{* * \dagger}$ \\
\hline BMI $\left(\mathrm{kg} / \mathrm{m}^{2}\right)$ & $23.4 \pm 0.5$ & $31.1 \pm 0.9^{* * *}$ & $29.8 \pm 1.3 * * *$ \\
\hline Fasting plasma glucose $(\mathrm{mmol} / \mathrm{l})$ & $5.5 \pm 0.1$ & $5.8 \pm 0.1$ & $8.9 \pm 0.7 * * * \dagger$ \\
\hline Fasting serum insulin (pmol/l) & $29.8 \pm 4$ & $39.6 \pm 6.1$ & $85.2 \pm 16.3 * *^{\dagger}$ \\
\hline $\mathrm{HbA}_{1 \mathrm{c}}(\%)$ & $5.5 \pm 0.1$ & $5.3 \pm 0.1$ & $6.8 \pm 0.4 * * * \dagger \dagger$ \\
\hline $\mathrm{HbA}_{1 \mathrm{c}}(\mathrm{mmol} / \mathrm{mol})$ & $36.2 \pm 1.0$ & $34.3 \pm 1.3$ & $51.2 \pm 3.9^{* * * \dagger \dagger}$ \\
\hline Plasma triacylglycerols $(\mathrm{mmol} / \mathrm{l})$ & $0.8 \pm 0.07$ & $1.0 \pm 0.3$ & $1.6 \pm 0.15^{* * * *^{\dagger \dagger}}$ \\
\hline GDR, basal (mg $\left.\min ^{-1} \mathrm{~m}^{-2}\right)$ & $75.7 \pm 2.6$ & $73.9 \pm 5.9$ & $72.9 \pm 3.3$ \\
\hline GDR, insulin $\left(\mathrm{mg} \min ^{-1} \mathrm{~m}^{-2}\right)$ & $380 \pm 24.9$ & $309 \pm 24 *$ & $160.1 \pm 25.7 * * * \dagger \dagger$ \\
\hline Diabetes duration (years) & - & - & $6.0 \pm 1.4$ \\
\hline
\end{tabular}

Data are presented as mean \pm SEM

$* p<0.05, * * p<0.01, * * * p<0.001$ vs lean control participants; ${ }^{\dagger} p<0.05,{ }^{\dagger \dagger} p<0.01,{ }^{\dagger \dagger} p<0.001$ vs overweight/obese control participants disposal rates (GDR) as previously described in detail [25, 40]. In study I, insulin was infused at a rate of $40 \mathrm{mU} \mathrm{m}^{-2} \mathrm{~min}^{-1}$ for $4 \mathrm{~h}$ [40]; whereas in study II, insulin was infused at a rate of $80 \mathrm{mU} \mathrm{m}^{-2} \mathrm{~min}^{-1}$ for $3 \mathrm{~h} \mathrm{[25].} \mathrm{In} \mathrm{both} \mathrm{studies,} \mathrm{the} \mathrm{clamp} \mathrm{was}$ preceded by a $2 \mathrm{~h}$ basal tracer $\left(3-\mathrm{H}^{3}\right.$-glucose) equilibration period. In study I, skeletal muscle biopsies were taken from the vastus lateralis muscle before (basal) and after (clamp) insulin infusion, while subcutaneous abdominal fat biopsies were obtained only before the clamp. Additional information on clamp and biopsies are given in ESM Methods.

Training programme (study II) Participants underwent 10 weeks of aerobic training as described previously [25, 42-44]. Briefly, the training programme consisted of 10 weeks of cycling on stationary bikes with four to five sessions of 20-35 min per week at an average exercise intensity of $\sim 65 \%$ of maximal oxygen consumption $\left(\mathrm{VO}_{2 \text { peak }}\right)$. For more information on the training programme, please refer to ESM Methods.

RNA isolation and cDNA synthesis For analysis of FGFR1c, FGFR3c, FGFR4 and KLB, RNA was isolated from $\sim 20 \mathrm{mg}$ human muscle tissue and resuspended in diethyl pyrocarbonate-treated $\mathrm{H}_{2} \mathrm{O}$ containing $0.1 \mathrm{mmol} / 1$ EDTA ( $1 \mu$ l per mg tissue). RNA from human fat was isolated from $\sim 30 \mathrm{mg}$ biopsy using the RNeasy mini kit (Qiagen, Venio, the Netherlands) following the manufacturer's instructions. cDNA was synthesised from $1 \mu \mathrm{g}$ skeletal muscle-derived RNA and $0.5 \mu \mathrm{g}$ WAT-derived RNA using an iScript cDNA synthesis kit (BioRad, Hercules, CA, USA). The amount of single-stranded DNA (ssDNA) was determined using Oligreen reagent (Molecular Probes, Leiden, the Netherlands) as previously described [45]. For analyses of the remaining genes, RNA isolation and cDNA synthesis from
10-20 mg wet muscle were performed as previously described [41] and as described in more detail in the ESM Methods.

qPCR analysis The cDNA of KLB, FGFR $1 c, F G F R 3 c$ and FGFR4 were amplified using LNA (locked nucleic acid) probes (Roche Life Science, Basel, Switzerland) and primers designed from human sequence data (Entrez-NIH and Ensembl, Sanger Institute, Cambridge, UK) using Primer Express software (Applied Biosystems, Foster City, CA, USA). The mRNA content of FGFR $1 c, 3 c$ and 4 was normalised to ssDNA as previously described [45]. For the remaining genes, quantitative realtime PCR (qPCR) was performed on an ABI Prism 7900HT sequence detection instrument using TaqMan custom Arrays (Applied Biosystems) according to the manufacturer's instructions. The data were analysed using qBasePlus software (Biogazelle, Zwijnaarde, Belgium). The mRNA levels in muscle were normalised to PPIA and $B 2 M$, while the transcript levels in adipose tissue were normalised to PPIA and ACTB. The expression of the reference genes did not differ between groups and were not affected by insulin. The mRNA abundances were calibrated to the sample with the lowest expression. The primer-probe pairs used are listed in ESM Table 2.

Serum FGF21 Serum FGF21 was measured using an ELISA kit (Biovendor, Brno, Czech Republic). The lowest detection level within the linear range in this ELISA is $7 \mathrm{pg} / \mathrm{ml}$ and values of serum FGF21 detected below this threshold were therefore all set to $7 \mathrm{pg} / \mathrm{ml}$. Both serum and plasma levels were measured without any detectable difference.

Statistical analysis The statistical analyses were performed using Sigma-Stat version 12.5 (Systat Software, San Jose, CA, USA). One-way or two-way ANOVA with repeated measures using Student-Newman-Keuls post hoc testing were 
used to evaluate differences between and within groups. Significance was accepted at $p<0.05$. Data are presented as mean $\pm \mathrm{SEM}$.

\section{Results}

Metabolic characteristics As previously reported [25, 40-42], fasting plasma glucose, plasma triacylglycerols, $\mathrm{HbA}_{1 \mathrm{c}}$ and serum insulin were elevated in the diabetic groups compared with the non-diabetic control groups in study I and II (Table 1 and ESM Table 1). Moreover, insulin-stimulated GDR was lower in individuals with type 2 diabetes compared with the control groups in study I and II, but it was also slightly lower in the overweight/obese group vs lean individuals in study I. In study II, exercise training increased insulinstimulated GDR in both groups, but the insulin-stimulated GDR was lower in individuals with type 2 diabetes both before and after training (ESM Table 1).

Serum FGF21 In study I, insulin increased serum FGF21 $(p<0.001)$ with no significant differences between groups (Fig. 1a). However, serum FGF21 in the basal state tended to be higher in individuals with type 2 diabetes when compared with both lean $(p=0.10)$ and overweight/obese $(p=0.057)$ control participants. In study II, insulin markedly increased serum FGF21 both before and after the training period in both groups $(p<0.001)$ (Fig. 1b). Furthermore, serum FGF21 was increased in individuals with type 2 diabetes compared with the overweight/obese control participants at all time points $(p<0.001)$ (Fig. 1b). There was no effect of exercise training on serum FGF21 in the groups, when measured at least $48 \mathrm{~h}$ after the last exercise session.

FGF21, its receptors and co-receptor in skeletal muscle In study I, insulin increased $F G F 21$ expression in muscle $(p=0.003)$ with no significant differences between groups
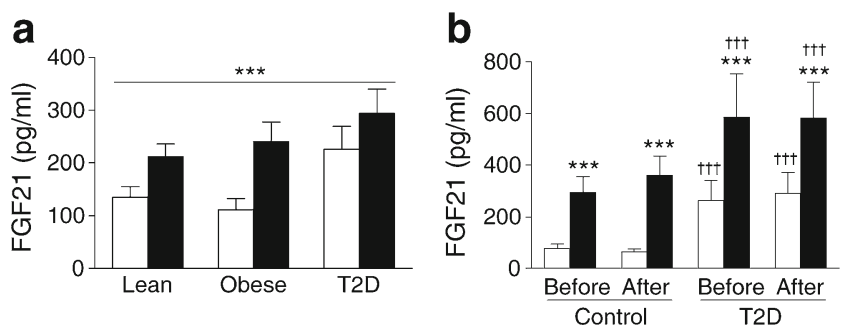

Fig. 1 Serum FGF21 in (a) lean $(n=12)$ and overweight/obese $(n=10)$ control participants and in individuals with type 2 diabetes (T2D, $n=9)$ in the basal (white bars) and insulin-stimulated state (black bars) of a euglycaemic-hyperinsulinaemic clamp, and (b) overweight/obese control individuals $(n=12)$ and individuals with type 2 diabetes $(n=12)$ in the basal (white bars) and insulin-stimulated state (black bars) of euglycaemic-hyperinsulinaemic clamps before and after a 10 week training intervention. Data are presented as mean \pm SEM. $* * * p<0.001$ vs basal (main effect in Fig. 1a); ${ }^{\dagger \dagger} p<0.001$ vs control participants
(Fig. 2a). However, when evaluated by one-way ANOVA analysis of basal values alone, we found increased FGF21 expression in muscle of individuals with type 2 diabetes compared with both lean $(p=0.026)$ and overweight/obese $(p=0.017)$ control participants. The insulin-mediated increase in FGF21 expression was not explained by increased expression of ATF4 in skeletal muscle (Fig. 2b). Expression of FGFR1c ( $p=0.005$ and $p=0.001$, respectively) and $F G F R 3 c$ ( $p=0.015$ and $p=0.018$, respectively) was reduced in muscle of individuals with type 2 diabetes compared with both lean and overweight/obese control participants (Fig. 2c, d), whereas the expression of FGFR4 was reduced only in individuals with type 2 diabetes $(p<0.004)$ compared with lean control participants (Fig. 2e). Expression of $K L B$ was either not detectable or hardly detectable from background (Ct-values $>35$ ) in human skeletal muscle (not shown).

FGF21, its receptors and co-receptor in adipose tissue In human WAT, expression of FGFR1c was increased in overweight/obese individuals $(p=0.005)$ and in individuals with type 2 diabetes $(p=0.003)$ compared with lean participants (Fig. 3a), whereas expression of $F G F R 3 c$ was similar between the groups (Fig. 3b). Expression of $K L B$ was reduced in overweight/obese control individuals $(p=0.002)$ and individuals with type 2 diabetes $(p=0.003)$ compared with lean participants (Fig. 3c). Transcript levels of FGF21 were not detectable in human WAT.

FGF21 target genes in human adipose tissue We then investigated whether the altered expression of FGFRIC and $K L B$ in overweight/obesity and type 2 diabetes was accompanied by changes in the expression of FGF 21 target genes. We observed no significant differences between groups in the expression of the genes encoding acetyl-CoA carboxylase 2 $(A C A C B)$, early growth response protein 1 (EGR1), leptin $(L E P)$, the hormone-sensitive lipase (LIPE), PEPCK1 $(P C K 1)$, adipose triglyceride lipase (PNPLA2), peroxisome proliferator-activated receptor gamma coactivator $1-\alpha$ (PPARGC1A), acyl-CoA desaturase (SCD) or GLUT1 (SLC2A1) (Fig. 4). FGF21 has been shown to promote browning of WAT by induction of brown adipocyte markers such $U C P 1$ and CIDEA [7, 9]. The expression of $U C P 1$ was very low in human WAT and could only be reliably detected in a few individuals (data not shown). The expression of CIDEA was, however, decreased in individuals with type 2 diabetes when compared with lean $(p=0.007)$ and overweight/obese $(p=0.014)$ control participants (Fig. 4c).

Correlation analyses In the total cohort of volunteers from study I $(n=32)$, serum FGF21 and muscle expression of FGF 21 correlated positively in both the basal $(r=0.651$, $p<0.001)$ and insulin-stimulated states $(r=0.535$, $p=0.003$ ), and when examining the increases induced by 


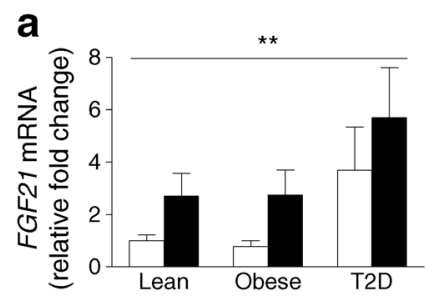

\section{b}
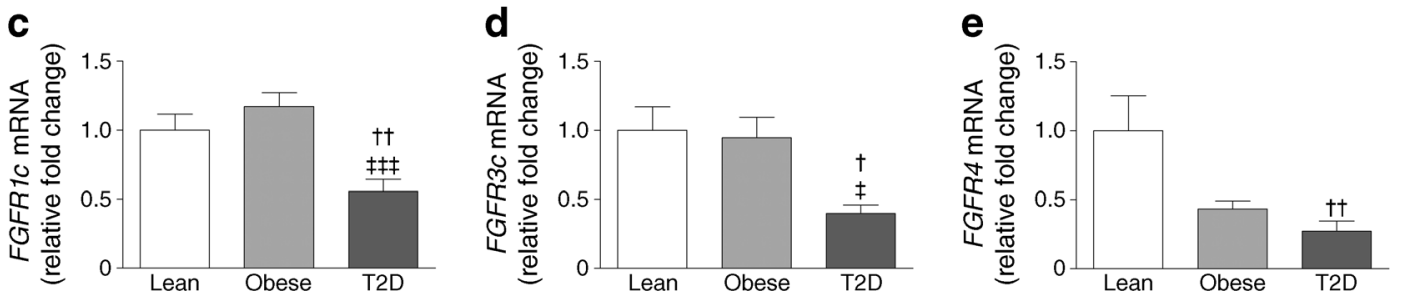

Fig. 2 mRNA levels of (a) FGF21 and (b) ATF4 in skeletal muscle of lean and overweight/obese control participants and in individuals with type 2 diabetes (T2D) in the basal (white bars) and insulin-stimulated state (black bars) of a euglycaemic-hyperinsulinaemic clamp. mRNA levels of (c) FGFR1c, (d) FGFR3c and (e) FGFR4 in skeletal muscle

of lean and overweight/obese participants and in individuals with type 2 diabetes in the basal state $(n=8-10)$. Data are presented as mean \pm SEM. $* * p<0.01 \mathrm{vs}$ basal (main effect); ${ }^{\dagger} p<0.05,{ }^{\dagger \dagger} p<0.01$ vs lean control participants; ${ }^{\ddagger} p<0.05,{ }^{\dagger+} p<0.001$ vs overweight/obese control participants

insulin ( $\Delta$ values) $(r=0.631, p<0.001)$ (Fig. 5a). Additionally, we found a positive correlation between serum FGF21 in the basal state and fasting insulin $(r=0.482, p=0.006)$ (Fig. 5b), $\mathrm{HbA}_{1 \mathrm{c}}(r=0.367, p=0.042)$ and triacylglycerol levels $(r=0.646, p<0.001)$, but not with BMI. Consistently, serum FGF21 in the basal state correlated negatively with insulinstimulated GDR $(r=-0.406, p=0.024)$. While $\mathrm{HbA}_{1 \mathrm{c}}$ was inversely related to expression of both FGFR1c $(r=-0.577$, $p=0.002$; Fig. 5c), FGFR3c $(r=-0.439, p=0.025)$ and FGFR4 $(r=-0.413, p=0.032)$ in skeletal muscle, BMI only correlated inversely with the expression of FGFR4 $(r=-0.385$, $p=0.048$ ). Finally, we identified a negative correlation between expression of $K L B$ in WAT and plasma triacylglycerols $(r=-0.420, p=0.021)$ and BMI $(r=-0.385, p=0.036)$ (Fig. 5d). The expression of FGFR1c in WAT did not correlate with BMI, and expression of $K L B$ or FGFR1c in WAT did not correlate with $\mathrm{HbA}_{1 \mathrm{c}}$ or fasting insulin. Expression of $C I D E A$ in WAT did not correlate with serum FGF21, but it correlated positively with insulin-stimulated GDR $(r=0.428, p=0.042)$ and inversely with BMI $(r=-0.519, p=0.011)$, fasting insulin $(r=-0.588, p=0.003), \mathrm{HbA}_{1 \mathrm{c}}(r=-0.463, p=0.026)$ and plasma triacylglycerols $(r=-0.488, p=0.018)$.

\section{Discussion}

In this study, we examined the effect of insulin and endurance training on serum FGF21, the effect of insulin on the expression of FGF21 and its putative receptors in human skeletal muscle, and the influence of overweight/obesity and type 2 diabetes on these responses, as well as on the mRNA levels of FGFRs and known FGF21 target genes in human WAT. While training had no effect on serum FGF21, we demonstrate that insulin within the physiological range increased serum FGF21 and the expression of FGF21 in human skeletal muscle, and that these responses are strongly correlated and, in contrast to our hypothesis, preserved in overweight/obesity and type 2 diabetes. Additionally, the expression of $K L B$ (encoding $\beta$-Klotho), was not detectable in human skeletal muscle, indicating that this is not an autocrine target tissue of FGF21. A novel finding is that mRNA levels of FGFRs appear to be regulated in a tissuespecific manner with reduced expression of FGFRIc, FGFR $3 c$ and FGFR4 in skeletal muscle of individuals with type 2 diabetes, while the expression of FGFRIc in WAT was markedly increased in both overweight/obesity and type 2 diabetes. The latter was accompanied by lower expression of $K L B$ in WAT, but
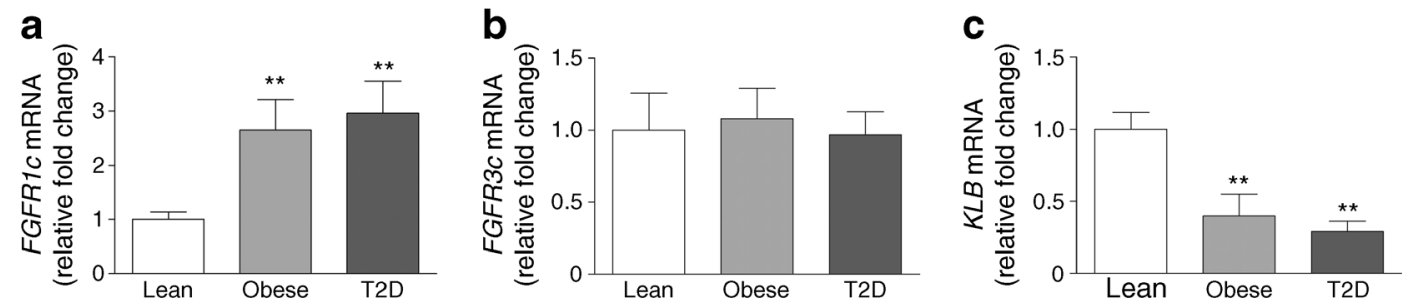

Fig. 3 Basal mRNA levels of (a) FGFR1c, (b) FGFR3c and (c) KLB in subcutaneous adipose tissue of lean $(n=6-8)$ and overweight/obese $(n=9-10)$ control participants and individuals with $\mathrm{T} 2 \mathrm{D}, n=12$. Data are presented as mean $\pm \mathrm{SEM}$. $* * p<0.01$ vs lean control participants 
a

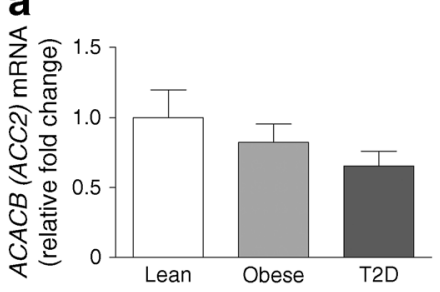

d

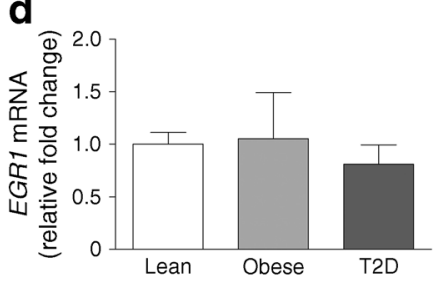

g
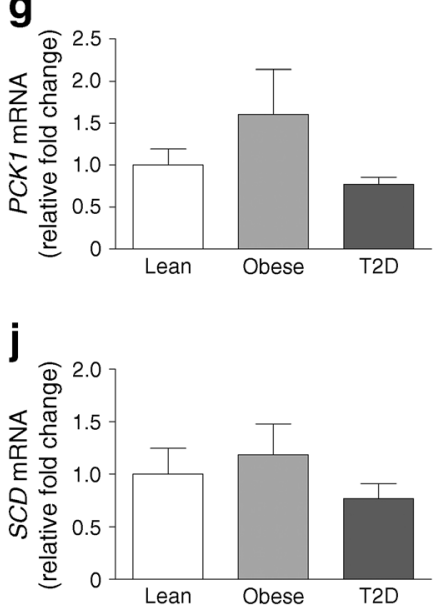

b

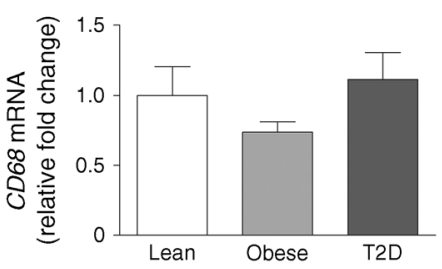

e

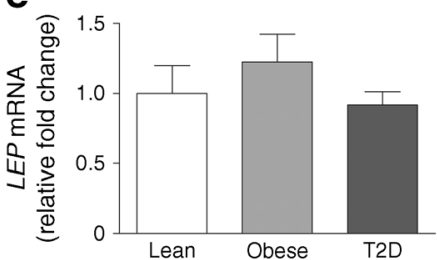

h

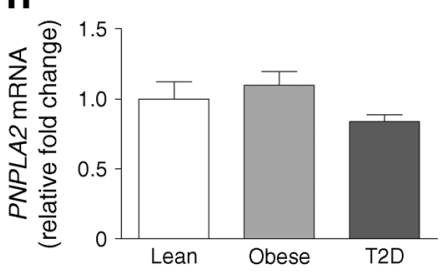

k

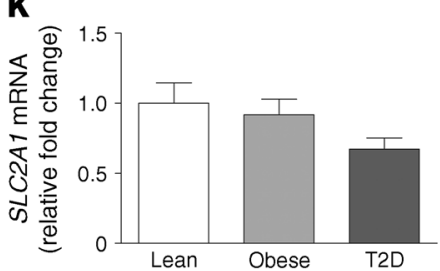

C

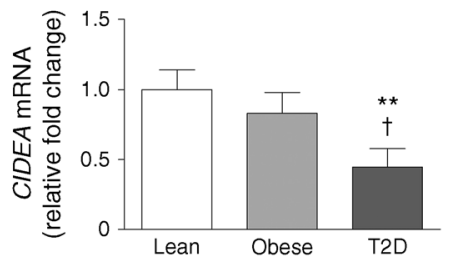

f

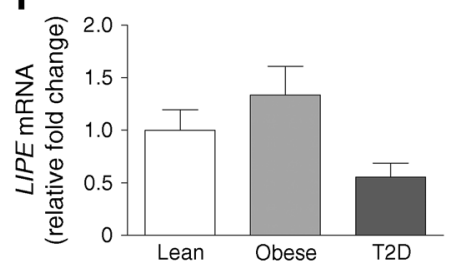

i

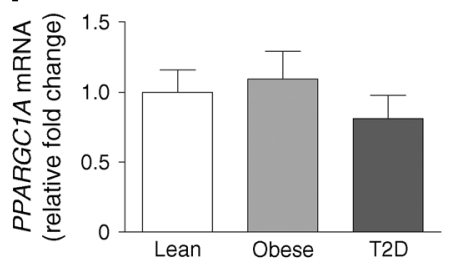

Fig. 4 Basal mRNA levels of (a) ACACB, (b) CD68, (c) CIDEA, (d) EGR1, (e) LEP, (f) LIPE, (g) PCK1, (h) PNPLA2, (i) PPARGC1A, (j) $S C D$ and (k) $S L C 2 A 1$ in subcutaneous adipose tissue of lean $(n=8)$ and overweight/obese $(n=8)$ control participants and individuals with T2D $n=7$. Data are presented as mean \pm SEM. $* * p<0.01$ vs lean control participants; ${ }^{\dagger} p<0.05$ vs overweight/obese control participants with no apparent differences in several FGF21 target genes. This is inconsistent with findings in mice, that have shown reduced expression of both KLB and FGFR1 in WAT of obese mice [39]. Our findings demonstrate that serum FGF21 and expression of its receptors in muscle and WAT are influenced by obesity and type 2 diabetes, and suggest that the presence of FGF21 resistance in obesity in humans could be due to reduced expression of $K L B$, but also that this may be compensated for through increased expression of FGFRIC or other mechanisms.

Several studies have reported increased serum FGF21 levels in individuals with obesity, the metabolic syndrome and type 2 diabetes [21, 35, 36, 38, 46]. In our training study (study II), serum FGF21 was significantly higher in individuals with type 2 diabetes compared with weight-matched control participants. A similar tendency for higher serum FGF21 levels in the basal state was observed in type 2 diabetes in study I. In agreement with previous studies [21, 22], our findings suggest that insulin resistance or other metabolic changes associated with type 2 diabetes rather than obesity per se cause increased serum FGF21. Interestingly, in the present study we observed that the serum FGF21 levels in the insulin-stimulated state of study II ( $3 \mathrm{~h}$ insulin infusion rate of $80 \mathrm{mU} \mathrm{m}^{-2} \mathrm{~min}^{-1}$ ) were $\sim 1.5-2.0$-fold higher in individuals with type 2 diabetes and matched overweight/obese individuals than the levels measured in the comparable groups in study I ( $4 \mathrm{~h}$ insulin infusion rate of $40 \mathrm{mU} \mathrm{m}^{-2} \mathrm{~min}^{-1}$ ). This indicates that the insulin-mediated induction of circulating FGF21 is dose-dependent in humans.

Several tissues, including skeletal muscle, may contribute to circulating FGF21 $[9,11,12,20]$, although serum FGF2 1 in response to fasting and diet-induced obesity is believed to be almost completely derived from the liver [14]. The first study identifying FGF21 as a potential myokine showed increased mRNA levels and secretion of FGF21 from skeletal muscle in Akt1-overexpressing mice [20]. In accordance, previous studies have reported that insulin promotes $F G F 21$ expression and release in murine and human muscle cells [20,21], as well as in muscle and serum of healthy young individuals $[20,22$, 


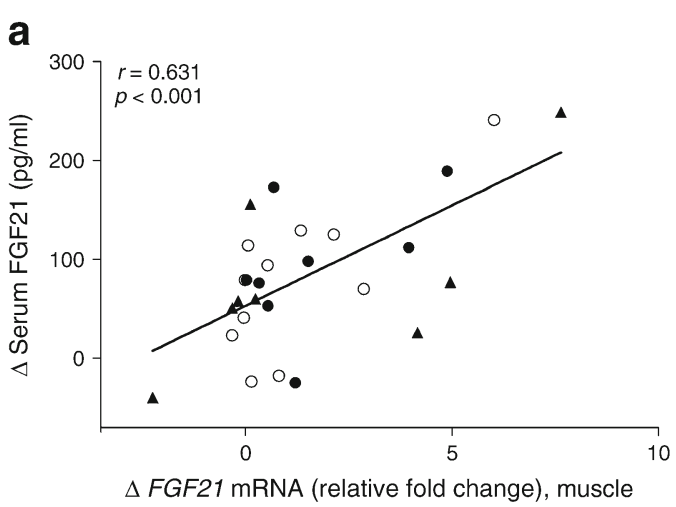

C

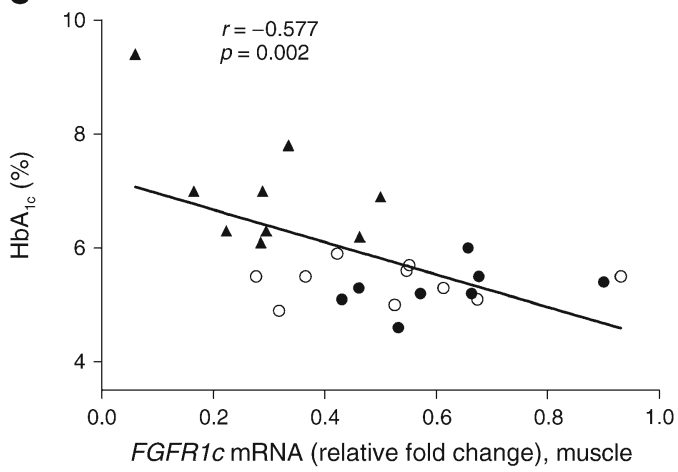

Fig. 5 The relationship between (a) insulin-mediated changes $(\Delta)$ in serum FGF21 and muscle $F G F 21$ mRNA levels; (b) fasting insulin and serum FGF21; (c) $\mathrm{HbA}_{1 \mathrm{c}}$ and muscle FGFRlc mRNA levels; and (d)

23]. In the present work, we investigated, for the first time, whether these responses to insulin were preserved in overweight/obesity and type 2 diabetes. We found that insulin markedly increased serum FGF21 in both studies and significantly increased muscle expression of $F G F 21$ with no impairment in overweight/obesity or type 2 diabetes in study I. Moreover, we observed a strong correlation between serum FGF21 and muscle expression of FGF21 in both the basal and insulin-stimulated states, and most convincingly also between the incremental increase in serum FGF21 and muscle FGF21 in response to insulin. These strong correlations lend support to the hypothesis that muscle FGF21, under certain conditions, is released to the circulation as a myokine, although further studies are needed to prove a causal relationship. It was recently shown that increased serum FGF21 after acute exercise was accompanied by an augmented release of FGF2 1 from the liver, and this appeared to be mediated by an elevated glucagon-to-insulin ratio [28]. Although, the pronounced increase in serum FGF21 observed in response to insulin infusion in our studies cannot be attributed to an elevated glucagon-to-insulin ratio, we cannot exclude that the hepatic expression and release of FGF21 are also induced by 3-4 h elevation of insulin, and quantitatively could play a larger role for the increase in serum FGF21 than release of FGF21 from the muscle.

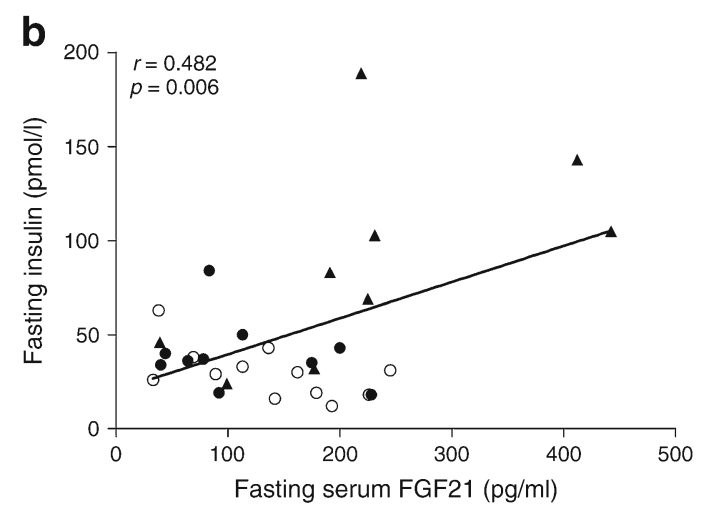

d

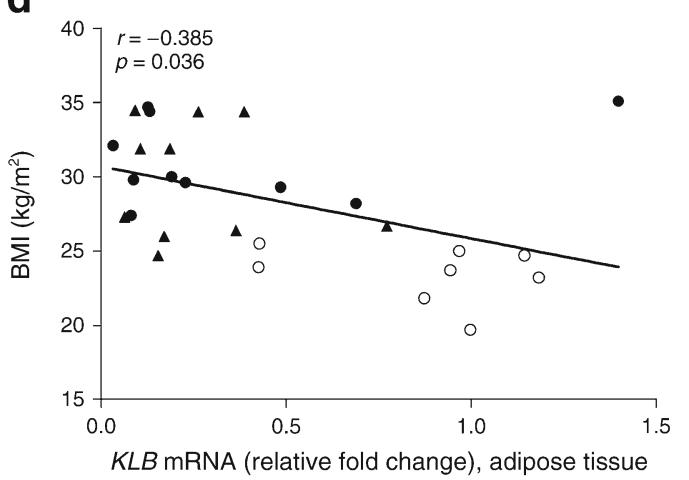

BMI and adipose tissue $K L B$ mRNA levels in a mixed population ( $n=27-32)$ of lean (white circles) and overweight/obese (black circles) control participants and individuals with type 2 diabetes (black triangles)

FGF21 signalling through the FGFRs requires $\beta$-Klotho as co-receptor $[15,16]$. To gain insight into the influence of obesity and type 2 diabetes on FGF21 signalling, we investigated the expression of $K L B, F G F R 1 c$ and $F G F R 3 c$, as well as the FGF19 receptor, FGFR4 in muscle of individuals with type 2 diabetes and matched overweight/obese and lean control participants. The expression of FGFRI $c$ and $F G F R 3 c$ was significantly lower in muscle from individuals with type 2 diabetes compared with both lean and overweight/obese individuals, while the expression of FGFR4 in muscle was reduced in both overweight/obese individuals and in individuals with type 2 diabetes compared with lean individuals. This suggests that FGFR-mediated signalling in skeletal muscle is affected by type 2 diabetes and insulin resistance, which is consistent with a recent study reporting reduced FGF signalling in skeletal muscle of non-obese individuals with impaired glucose tolerance or type 2 diabetes [46]. However, mRNA levels of $K L B$ in muscle were not detectable. This finding confirms previous reports of a limited $\beta$-Klotho expression in muscle $[18,21]$ and provides evidence that skeletal muscle is not an autocrine target tissue of FGF21 in humans. Further studies are needed to address the potential consequences of reduced FGFR expression in type 2 diabetes and overweight/ obesity. Of interest, a recent report showed that pharmacological doses of human recombinant FGF1 promotes insulin- 
dependent glucose uptake in skeletal muscle, and that this glucose-lowering effect of FGF1 was mediated primarily via FGFR1 signalling [47]. Thus, reduced FGFRIc mRNA levels could play a role in insulin resistance in muscle of individuals with type 2 diabetes.

Several studies have suggested that reduced $\beta$-Klotho levels in adipose tissue may confer FGF21 resistance in obesity [37, $39,48]$. Interestingly, we found reduced $K L B$ expression, while the expression of $F G F R 1$ was markedly increased in subcutaneous WAT in overweight/obese and type 2 diabetes individuals. Thus, our data confirms previous findings of reduced $\beta$-Klotho in WAT of obese mice [39], high-fat-fed nonhuman primates [48] and obese humans with varying degrees of abnormal glucose homeostasis [37]. These data indicate that FGF21 signalling through $\beta$-Klotho in WAT is affected primarily by obesity rather than the altered metabolic control associated with type 2 diabetes. FGFR1c has been suggested as the predominant FGFR involved in FGF21 signalling [15]. Our results suggest the possibility that FGFR $1 c$ expression is increased to compensate for reduced $K L B$ expression, potentially in a $\beta$-Klotho-independent manner [47]. This is supported by the finding that the expression of several FGF21 target genes was unaffected by overweight/obesity and type 2 diabetes. While further studies are needed to clarify the mechanisms, our results from human WAT and muscle indicate that FGFRmediated signalling is regulated by metabolic challenges in a tissue-specific manner. Thus, increased $\mathrm{HbA}_{1 \mathrm{c}}$ appears to be a major cause of reduced FGFR expression in muscle of individuals with type 2 diabetes, while overweight/obesity appears to explain lower expression of $K L B$ in human WAT.

FGF21 has been reported to promote lipolysis, GLUT1mediated glucose uptake and browning of WAT $[1,7,9,11$, 21]. In humans, the effect of physiological concentrations of FGF21 on WAT is not clear. We investigated whether the changes in serum FGF21 and expression of KLB and FGFR1 in WAT in overweight/obesity and type 2 diabetes were accompanied with altered expression of known FGF21 target genes. However, we did not observe any influence of overweight/ obesity or type 2 diabetes on the expression of genes involved in the early gene response (EGR1), glucose uptake (SLC2A1), lipid metabolism (ACACB, LIPE, PCK1, PNPLA2, SCD) or mitochondrial biogenesis $(P P A R G C 1 A)[1,11,21]$ or in the genes encoding leptin or CD68, of which the latter is a marker of macrophage infiltration. Several studies have suggested that FGF21 increases energy expenditure through browning of WAT [7-9]. While expression of UCP1 was only detectable in a few individuals (data not shown), we demonstrated decreased expression of CIDEA in individuals with type 2 diabetes. Moreover, expression of CIDEA in WAT correlated positively with insulin sensitivity and negatively with $\mathrm{BMI}, \mathrm{HbA}_{1 \mathrm{c}}$, fasting insulin and triacylglycerols, but we did not observe any relationship with serum FGF21. To our knowledge, this has not been reported previously. However, in morbidly obese individuals, expression of CIDEA was reduced twofold in WAT, but was normalised after weight reduction following gastric bypass surgery $[49,50]$. Moreover, in agreement with our findings, expression of CIDEA in adipose tissue was inversely related to several markers for metabolic health, including HOMA-IR, as a measure of insulin resistance, and waist circumference [49, 50].

Regular physical activity protects against several chronic conditions including obesity, type 2 diabetes and cardiovascular disease $[24,25]$. Studies of the effect of exercise training on circulating FGF21 has yielded inconsistent findings with both increased [21,32], reduced [30,33], or no changes in circulating FGF21 [31]. The diverse responses are probably explained by different durations, intensity and modes of exercise interventions, as well as differences in the study cohorts. Consistent with findings of Besse-Patin et al in a cohort of obese men [31], we demonstrate that 10 weeks of endurance training does not change serum FGF21 in individuals with type 2 diabetes or glucose tolerant weight-matched individuals despite significant improvements (13-22\%) in insulin sensitivity measured as insulin-stimulated GDR as reported previously [42]. Furthermore, exercise training did not alter the response of serum FGF21 to insulin administration, suggesting that the ability of insulin to increase serum FGF21 is dissociated from the changes in insulin action on peripheral glucose uptake.

In summary, we have shown that physiological insulin concentrations induce muscle expression of FGF21 and that this correlates strongly with the increase in serum FGF21 in humans. These responses appear to be preserved in overweight/obesity and type 2 diabetes. Moreover, we found elevated serum and muscle FGF21 levels in individuals with type 2 diabetes. In adipose tissue of overweight/obese individuals with or without type 2 diabetes, the expression of $K L B$ (encoding $\beta$-Klotho) was reduced suggesting that obesity in humans is a state of FGF21 resistance. However, this may be compensated for by increased expression of FGFR $1 c$ or other mechanisms, which seem to rescue FGF21 signalling at least as evaluated by the unaffected expression of most FGF21 target genes. Further studies are needed to understand the potential role of FGF21 as a myokine and the potential existence and consequences of FGF21 resistance in humans.

Acknowledgements We would like to thank L. Hansen and C.B. Olsen of the Department of Endocrinology, Odense University Hospital, and A.S. Pedersen of the Department of Clinical Research, University of Southern Denmark for their skilled technical assistance.

Data availability The data are available on request.

Funding This study was supported by grants from the Danish Council for Independent Research (Sapere Aude, DFF Starting Grant) and from the Novo Nordisk Foundation. 
Duality of interest SGV and BA are employees of Novo Nordisk. The remaining authors confirm that there is no duality of interest associated with this manuscript.

Contribution statement $\mathrm{KH}$ and BA wrote the study protocol. BFV identified the eligible participants and conducted the intervention study, thereby acquiring the metabolic data as well as material for the laboratory experiments. SGV and RK conducted the laboratory experiments and analysed the data. RK and $\mathrm{KH}$ wrote the manuscript. All authors critically revised the paper and approved the final version of the manuscript. $\mathrm{KH}$ is the guarantor of this work and, as such, had full access to all the data in the study and takes full responsibility for the integrity of the data and the accuracy of the data analysis.

\section{References}

1. Kharitonenkov A, Shiyanova TL, Koester A et al (2005) FGF-21 as a novel metabolic regulator. J Clin Invest 115:1627-1635

2. Wente W, Efanov AM, Brenner M et al (2005) Fibroblast growth factor-21 improves pancreatic beta-cell function and survival by activation of extracellular signal-regulated kinase 1/2 and Akt signaling pathways. Diabetes 55:2470-2478

3. Kharitonenkov A, Wroblewski VJ, Koester A et al (2007) The metabolic state of diabetic monkeys is regulated by fibroblast growth factor-21. Endocrinology 148:774-781

4. Coskun T, Bina HA, Schneider MA et al (2008) Fibroblast growth factor 21 corrects obesity in mice. Endocrinology 149:6018-6027

5. Xu J, Lloyd DJ, Hale C et al (2009) Fibroblast growth factor 21 reverses hepatic steatosis, increases energy expenditure, and improves insulin sensitivity in diet-induced obese mice. Diabetes 58: 250-259

6. Ding X, Boney-Montoya J, Owen BM et al (2012) BetaKlotho is required for fibroblast growth factor 21 effects on growth and metabolism. Cell Metab 16:387-393

7. Fisher FM, Kleiner S, Douris N et al (2012) FGF21 regulates PGC1 alpha and browning of white adipose tissues in adaptive thermogenesis. Genes Dev 26:271-281

8. Ost M, Coleman V, Voigt A et al (2016) Muscle mitochondrial stress adaptation operates independently of endogenous FGF21 action. Mol Metab 5:79-90

9. Kim KH, Jeong YT, Oh H et al (2013) Autophagy deficiency leads to protection from obesity and insulin resistance by inducing Fgf21 as a mitokine. Nat Med 19:83-92

10. Lee MS, Choi SE, Ha ES et al (2012) Fibroblast growth factor-21 protects human skeletal muscle myotubes from palmitate-induced insulin resistance by inhibiting stress kinase and NF-kappaB. Metabolism 61:1142-1151

11. Badman MK, Pissios P, Kennedy AR, Koukos G, Flier JS, MaratosFlier E (2007) Hepatic fibroblast growth factor 21 is regulated by PPARalpha and is a key mediator of hepatic lipid metabolism in ketotic states. Cell Metab 5:426-437

12. Inagaki T, Dutchak $P$, Zhao G et al (2007) Endocrine regulation of the fasting response by PPARalpha-mediated induction of fibroblast growth factor 21. Cell Metab 5:415-425

13. Owen BM, Mangelsdorf DJ, Kliewer SA (2015) Tissue-specific actions of the metabolic hormones FGF15/19 and FGF21. Trends Endocrinol Metab 26:22-29

14. Markan KR, Naber MC, Ameka MK et al (2014) Circulating FGF21 is liver derived and enhances glucose uptake during refeeding and overfeeding. Diabetes 63:4057-4063

15. Kurosu H, Choi M, Ogawa Y et al (2007) Tissue-specific expression of betaKlotho and fibroblast growth factor (FGF) receptor isoforms determines metabolic activity of FGF19 and FGF21. J Biol Chem 282:26687-26695

16. Ogawa Y, Kurosu H, Yamamoto M et al (2007) BetaKlotho is required for metabolic activity of fibroblast growth factor 21. Proc Natl Acad Sci U S A 104:7432-7437

17. Ito S, Kinoshita S, Shiraishi $\mathrm{N}$ et al (2000) Molecular cloning and expression analyses of mouse betaklotho, which encodes a novel Klotho family protein. Mech Dev 98:115-119

18. Fon Tacer K, Bookout AL, Ding X et al (2010) Research resource: comprehensive expression atlas of the fibroblast growth factor system in adult mouse. Mol Endocrinol 24:2050-2064

19. Tyynismaa H, Carroll CJ, Raimundo N et al (2010) Mitochondrial myopathy induces a starvation-like response. Hum Mol Genet 19: 3948-3958

20. Izumiya Y, Bina HA, Ouchi N, Akasaki Y, Kharitonenkov A, Walsh $\mathrm{K}$ (2008) FGF21 is an Akt-regulated myokine. FEBS Lett 582: $3805-3810$

21. Mashili FL, Austin RL, Deshmukh AS et al (2011) Direct effects of FGF21 on glucose uptake in human skeletal muscle: implications for type 2 diabetes and obesity. Diabetes Metab Res Rev 27:286-297

22. Hojman P, Pedersen M, Nielsen AR et al (2009) Fibroblast growth factor-21 is induced in human skeletal muscles by hyperinsulinemia. Diabetes 58:2797-2801

23. Vienberg SG, Brons C, Nilsson E, Astrup A, Vaag A, Andersen B (2012) Impact of short-term high-fat feeding and insulin-stimulated FGF21 levels in subjects with low birth weight and controls. Eur J Endocrinol 167:49-57

24. Knowler WC, Barrett-Connor E, Fowler SE et al (2002) Reduction in the incidence of type 2 diabetes with lifestyle intervention or metformin. N Engl J Med 346:393-403

25. Vind BF, Pehmoller C, Treebak JT et al (2011) Impaired insulininduced site-specific phosphorylation of TBC1 domain family, member 4 (TBC1D4) in skeletal muscle of type 2 diabetes patients is restored by endurance exercise-training. Diabetologia 54:157-167

26. Schnyder S, Handschin C (2015) Skeletal muscle as an endocrine organ: PGC-1alpha, myokines and exercise. Bone 80:115-125

27. Kim KH, Kim SH, Min YK, Yang HM, Lee JB, Lee MS (2013) Acute exercise induces FGF21 expression in mice and in healthy humans. PLoS One 8:e63517

28. Hansen JS, Clemmesen JO, Secher NH et al (2015) Glucagon-toinsulin ratio is pivotal for splanchnic regulation of FGF-21 in humans. Mol Metab 4:551-560

29. Hansen JS, Pedersen BK, Xu G et al (2016) Exercise-induced secretion of FGF21 and follistatin are blocked by pancreatic clamp and impaired in type 2 diabetes. J Clin Endocrinol Metab 101: 2816-2285

30. Yang SJ, Hong HC, Choi HY et al (2011) Effects of a three-month combined exercise programme on fibroblast growth factor 21 and fetuin-A levels and arterial stiffness in obese women. Clin Endocrinol 75:464-469

31. Besse-Patin A, Montastier E, Vinel C et al (2014) Effect of endurance training on skeletal muscle myokine expression in obese men: identification of apelin as a novel myokine. Int J Obes 38:707-713

32. Cuevas-Ramos D, Almeda-Valdes P, Meza-Arana CE et al (2012) Exercise increases serum fibroblast growth factor 21 (FGF21) levels. PLoS One 7:e38022

33. Taniguchi H, Tanisawa K, Sun X, Kubo T, Higuchi M (2015) Endurance exercise reduces hepatic fat content and serum fibroblast growth factor 21 levels in elderly men. J Clin Endocrinol Metab 101:191-198

34. Galman C, Lundasen T, Kharitonenkov A et al (2008) The circulating metabolic regulator FGF21 is induced by prolonged fasting and PPARalpha activation in man. Cell Metab 8:169-174

35. Zhang X, Yeung DC, Karpisek M et al (2008) Serum FGF21 levels are increased in obesity and are independently associated with the metabolic syndrome in humans. Diabetes 57:1246-1253 
36. Chavez AO, Molina-Carrion M, Abdul-Ghani MA, Folli F, DeFronzo RA, Tripathy D (2009) Circulating fibroblast growth factor- 21 is elevated in impaired glucose tolerance and type 2 diabetes and correlates with muscle and hepatic insulin resistance. Diabetes Care 32:1542-1546

37. Gallego-Escuredo JM, Gomez-Ambrosi J, Catalan V, Domingo P, Giralt M, Fruhbeck G (2015) Opposite alterations in FGF21 and FGF19 levels and disturbed expression of the receptor machinery for endocrine FGFs in obese patients. Int J Obes 39:121-129

38. Cheng X, Zhu B, Jiang F, Fan H (2011) Serum FGF-21 levels in type 2 diabetic patients. Endocr Res 36:142-148

39. Fisher FM, Chui PC, Antonellis PJ et al (2010) Obesity is a fibroblast growth factor 21 (FGF21)-resistant state. Diabetes 59:2781-2789

40. Vind BF, Birk JB, Vienberg SG et al (2012) Hyperglycaemia normalises insulin action on glucose metabolism but not the impaired activation of AKT and glycogen synthase in the skeletal muscle of patients with type 2 diabetes. Diabetologia 55:1435-1445

41. Kruse R, Vind BF, Petersson SJ, Kristensen JM, Hojlund K (2015) Markers of autophagy are adapted to hyperglycaemia in skeletal muscle in type 2 diabetes. Diabetologia 58:2087-2095

42. Hey-Mogensen M, Hojlund K, Vind BF et al (2010) Effect of physical training on mitochondrial respiration and reactive oxygen species release in skeletal muscle in patients with obesity and type 2 diabetes. Diabetologia 53:1976-1985

43. Mogensen M, Vind BF, Hojlund K, Beck-Nielsen H, Sahlin K (2009) Maximal lipid oxidation in patients with type 2 diabetes is normal and shows an adequate increase in response to aerobic training. Diabetes Obes Metab 11:874-883

44. Nielsen J, Mogensen M, Vind BF et al (2010) Increased subsarcolemmal lipids in type 2 diabetes: effect of training on localization of lipids, mitochondria, and glycogen in sedentary human skeletal muscle. Am J Physiol Endocrinol Metab 298:E706-E713

45. Lundby C, Nordsborg N, Kusuhara K, Kristensen KM, Neufer PD, Pilegaard H (2015) Gene expression in human skeletal muscle: alternative normalization method and effect of repeated biopsies. Eur J Appl Physiol 95:351-360

46. Jeon JY, Choi SE, Ha ES et al (2016) Association between insulin resistance and impairment of FGF21 signal transduction in skeletal muscles. Endocrine 53:97-196

47. Suh JM, Jonker JW, Ahmadian M et al (2014) Endocrinization of FGF1 produces a neomorphic and potent insulin sensitizer. Nature 513:436-439

48. Nygaard EB, Moller CL, Kievit P, Grove KL, Andersen B (2014) Increased fibroblast growth factor 21 expression in high-fat diet-sensitive non-human primates (Macaca mulatta). Int J Obes 38:183-191

49. Nordstrom EA, Ryden M, Backlund EC et al (2005) A humanspecific role of cell death-inducing DFFA (DNA fragmentation factor-alpha)-like effector A (CIDEA) in adipocyte lipolysis and obesity. Diabetes 54:1726-1734

50. Puri V, Ranjit S, Konda S et al (2008) Cidea is associated with lipid droplets and insulin sensitivity in humans. Proc Natl Acad Sci U S A 105:7833-7838 